\title{
CONFLICT-FREE CONNECTIONS OF GRAPHS
}

\author{
JÚLIUS CZAP \\ Department of Applied Mathematics and Business Informatics \\ Faculty of Economics, Technical University of Košice \\ Němcovej 32, 04001 Košice, Slovakia \\ e-mail: julius.czap@tuke.sk \\ STANISLAV JENDROL \\ AND \\ JURAJ VALISKa \\ Institute of Mathematics, P.J. Šafárik University \\ Jesenná 5, 04001 Košice, Slovakia \\ e-mail: stanislav.jendrol@upjs.sk \\ juraj.valiska@student.upjs.sk
}

\begin{abstract}
An edge-colored graph $G$ is conflict-free connected if any two of its vertices are connected by a path, which contains a color used on exactly one of its edges. In this paper the question for the smallest number of colors needed for a coloring of edges of $G$ in order to make it conflict-free connected is investigated. We show that the answer is easy for 2-edge-connected graphs and very difficult for other connected graphs, including trees.
\end{abstract}

Keywords: edge-coloring, conflict-free connection, 2-edge-connected graph, tree.

2010 Mathematics Subject Classification: 05C15.

\section{REFERENCES}

[1] S.A. van Aardt, C. Brause, A.P. Burger, M. Frick, A. Kemnitz and I. Schiermeyer, Proper connection and size of graphs, Discrete Math. 340 (2017) 2673-2677. doi:10.1016/j.disc.2016.09.021 
[2] E. Andrews, C. Lumduanhom, E. Laforge and P. Zhang, On proper-path colorings in graphs, J. Combin. Math. Combin. Comput. 97 (2016) 189-207.

[3] V. Borozan, S. Fujita, A. Gerek, C. Magnant, Y. Manoussakis, L. Montero and Zs. Tuza, Proper connection of graphs, Discrete Math. 312 (2012) 2550-2560. doi:10.1016/j.disc.2011.09.003

[4] G. Chartrand, G.L. Johns, K.A. McKeon and P. Zhang, Rainbow connection in graphs, Math. Bohem. 133 (2008) 85-98.

[5] G. Chartrand, L. Lesniak and P. Zhang, Graphs and Digraphs, Sixth Edition (Boca Raton, CRC Press, 2016).

[6] P. Cheilaris, B. Keszegh and D. Pálvölgyi, Unique-maximum and conflict-free coloring for hypergraphs and tree graphs, SIAM J. Discrete Math. 27 (2013) 1775-1787. doi:10.1137/120880471

[7] P. Cheilaris and G. Tóth, Graph unique-maximum and conflict-free colorings, J. Discrete Algorithms 9 (2011) 241-251.

doi:10.1016/j.jda.2011.03.005

[8] I. Fabrici and F. Göring, Unique-maximum coloring of plane graphs, Discuss. Math. Graph Theory 36 (2016) 95-102.

doi:10.7151/dmgt.1846

[9] R. Gu, X. Li and Z. Qin, Proper connection number of random graphs, Theoret. Comput. Sci. 609 (2016) 336-343.

doi:10.1016/j.tcs.2015.10.017

[10] F. Huang, X. Li and S. Wang, Proper connection number and 2-proper connection number of a graph. arxiv.org/pdf/1507.01426v2.pdf

[11] N. Kamčev, M. Krivelevich and B. Sudakov, Some remarks on rainbow connectivity, J. Graph Theory 83 (2016) 372-383. doi: $10.1002 /$ jgt.22003

[12] A. Kemnitz, J. Przybyło, I. Schiermeyer and M. Woźniak, Rainbow connection in sparse graphs, Discuss. Math. Graph Theory 33 (2013) 181-192. doi:10.7151/dmgt.1640

[13] A. Kemnitz and I. Schiermeyer, Graphs with rainbow connection number two, Discuss. Math. Graph Theory 31 (2011) 313-320. doi:10.7151/dmgt.1547

[14] X. Li, M. Liu and I. Schiermeyer, Rainbow connection number of dense graphs, Discuss. Math. Graph Theory 33 (2013) 603-611.

doi:10.7151/dmgt.1692

[15] X. Li and C. Magnant, Properly colored notions of connectivity - a dynamic survey, Theory and Applications of Graphs 0, Article 2 (2015).

doi:10.20429/tag.2015.000102 
[16] X. Li, Y. Shi and Y. Sun, Rainbow connections of graphs: a survey, Graphs Combin. 29 (2013) 1-38.

doi:10.1007/s00373-012-1243-2

[17] X. Li and Y. Sun, Rainbow Connections of Graphs (Springer Briefs in Mathematics, Berlin, Springer, 2012).

[18] K. Makino, Y. Uno and T. Ibaraki, On minimum edge ranking spanning trees, J. Algorithms 38 (2001) 411-437. doi:10.1006/jagm.2000.1143

[19] R. Melville and W. Goddard, Coloring graphs to produce properly colored walks, Graphs Combin. 33 (2017) 1271-1281. doi:10.1007/S00373-017-1843-y

[20] J. Pach and G. Tardos, Conflict-free colourings of graphs and hypergraphs, Comb. Probab. Comput. 18 (2009) 819-834. doi:10.1017/S0963548309990290

[21] I. Schiermeyer, Rainbow connection in graphs with minimum degree three, IWOCA 2009, Lecture Notes in Comput. Sci. 5874 (2009) 432-437. doi:10.1007/978-3-642-10217-2 42

[22] V.G. Vizing, On an estimate of the chromatic class of a p-graph, Diskret. Analiz. 3 (1964) 25-30.

[23] D.B. West, Introduction to Graph Theory, Second Edition (New Delhi, PrenticeHall, 2005).

Received 4 November 2016

Revised 3 March 2017

Accepted 3 March 2017 